Article

\title{
Identification of Metabolites of the Cardioprotective Alkaloid Dehydrocorydaline in Rat Plasma and Bile by Liquid Chromatography Coupled with Triple Quadrupole Linear Ion Trap Mass Spectrometry
}

\author{
Huanyu Guan ${ }^{1,2}$, Kaitong Li ${ }^{1}$, Xiaoming Wang ${ }^{1}$, Xiaomei Luo ${ }^{1}$, Meifeng Su ${ }^{1}$, Wenting Tan ${ }^{1}$, \\ Xiaoyan Chang ${ }^{1}$ and Yue Shi ${ }^{1, *}$ \\ 1 Institute of Medicinal Plant Development, Chinese Academy of Medical Sciences and Peking Union Medical \\ College, Beijing 100193, China; guanhuanyu630@163.com (H.G.); likaitong@joinn-lab.com (K.L.); \\ lmlwxm123@163.com (X.W.); Luoxiaomei1019@163.com (X.L.); 20160931861@bucm.edu.cn (M.S.); \\ 18811790612@163.com (W.T.); changxiaoyan1234@163.com (X.C.) \\ 2 School of Pharmaceutical Sciences, Guizhou Medical University, Guiyang 550004, China \\ * Correspondence: shiyue1029@126.com; Tel.: +86-10-5783-3255; Fax: +86-10-5783-3270
}

Received: 15 September 2017; Accepted: 3 October 2017; Published: 10 October 2017

\begin{abstract}
Dehydrocorydaline (DHC), a quaternary alkaloid from Corydalis yanhusuo, has been demonstrated to be the active constituent in the treatment of coronary heart disease. In this study, a high-performance liquid chromatography-electrospray ionization-triple quadrupole linear ion trap mass spectrometry (HPLC-ESI-QTRAP MS) technique was used to identify DHC metabolites in plasma and bile after oral administration of DHC to rats. A total of 18 metabolites (M1 to M18) were identified and characterized by LC-MS/MS in the positive ion mode. These 18 metabolites were all present in rat bile, while only 9 were detected in plasma. $O$-demethylation, hydroxylation, di-hydroxylation, glucuronidation of $O$-demethyl DHC, sulfation of $O$-demethyl DHC and di-hydroxylation of dehydro-DHC were the major metabolic pathways of DHC. This is the first time that these metabolites of DHC have been identified in rat plasma and bile, which provides useful information for further analysis of the biotransformation of DHC and other quaternary protoberberine-type alkaloids.
\end{abstract}

Keywords: LC-MS/MS; dehydrocorydaline; Corydalis yanhusuo; metabolites; cardioprotection

\section{Introduction}

Corydalis yanhusuo W.T. Wang, a perennial herb of the Papaveraceae family, is well known as a traditional Chinese medicine (TCM) [1]. The preparation of C. yanhusuo extract, as Ke-Da-Ling tablets, has been used to treat cardiovascular disease in modern clinical practice [2]. A quaternary protoberberine alkaloid of $C$. yanhusuo, namely, dehydrocorydaline (DHC), has been demonstrated to be the active constituent in the treatment of coronary heart disease [3]. Structurally, DHC is comprised of 5,6-dihydrodibenzo $[a, g]$ quinolizinium $\left(\mathrm{C}_{17} \mathrm{H}_{14} \mathrm{~N}^{+}\right)$[4], a methyl group at $\mathrm{C}-13$ and four methoxyl groups at C-2, $-3,-9$ and -10 . Pharmacological studies have revealed that DHC can improve hypoxia tolerance in rats, expand the coronary artery and increase the blood flow in this artery [5]. Through the previously established fingerprint-efficacy relationship between chemical fingerprints of quaternary ammonium alkaloids from C. yanhusuo and its cardioprotection efficiency, we found that DHC is one of the main effective components of this TCM and can be adopted as a reference component for quality control of C. yanhusuo [6]. In addition, DHC is also used as a reference index to evaluate the quality of Ke-Da-Ling tablets [7]. 
The in vivo metabolism of this drug plays an important role in the production of active metabolites and the generation of the resulting activities. Radioactive tracing experiments showed that DHC was subjected to the first-pass effect in the liver and was distributed mainly in the digestive tract, liver and kidney after oral administration of ${ }^{14} \mathrm{C}$-dehydrocorydaline to rats. The compound was metabolized by $\mathrm{O}$-demethylation and then by glucuronidation [8]. However, the metabolic fate of DHC was not completely established.

In our previous study, the in vivo metabolism of C. yanhusuo extract in rat plasma was analyzed by high-performance liquid chromatography coupled to mass spectrometry (HPLC-MS), which revealed that 10 bioactive components of $C$. yanhusuo were present in the blood [9]. Additionally, we conducted a comparative pharmacokinetic analysis of DHC after administration of C. yanhusuo and pure DHC using LC-MS/MS [10], and found that the oral administration of C. yanhusuo extract accelerated the absorption of DHC and slowed down its elimination.

With the development of liquid chromatography coupled with mass spectrometry (LC-MS) and data acquisition software, the identification of metabolites present at low concentrations in complex biological matrices has become easier to achieve. To further evaluate the in vivo metabolism of DHC and to obtain more information for pharmacological research, a high-performance liquid chromatography-electrospray ionization-triple quadrupole linear ion trap mass spectrometry (HPLC-ESI-QTRAP MS) method was developed to analyze the metabolites of DHC in rat plasma and bile. A total of 18 metabolites were identified using the multiple reaction monitoring-information-dependent acquisition-triggering enhanced product ion (MRM-IDA-EPI) mode.

\section{Results and Discussion}

\subsection{Optimization of HPLC Conditions}

To improve the resolution and intensities of chromatographic peaks, various compositions of the mobile phase (methanol, acetonitrile, ammonium acetate and formic acid) were tested to optimize the chromatographic conditions. As a result, acetonitrile and water (containing $0.8 \%$ formic acid and $10 \mathrm{mM}$ ammonium acetate; 25:75 $\mathrm{v} / \mathrm{v}$ ) were employed as the mobile phase because of the best separation and an abundant signal.

\subsection{Optimization of Sample Preparation}

Liquid-liquid extraction (ethyl acetate and water-saturated $n$-butanol), protein precipitation (acetone, acetonitrile and methanol) and solid-phase extraction (SPE) were evaluated to obtain the metabolites. Compared with liquid-liquid extraction, SPE could ensure the simultaneous extraction of target compounds. The higher intensities of target peaks were obtained via SPE compared to using protein precipitation. Moreover, methanol containing $2 \%$ formic acid was selected as the elution solvent for SPE. To determine the optimum volume of methanol containing $2 \%$ formic acid to elute target compounds from the SPE cartridge, the elution was carried out two times with $1.0 \mathrm{~mL}$ of methanol containing $2 \%$ formic acid for each time. It was concluded that $1.0 \mathrm{~mL}$ of methanol containing $2 \%$ formic acid was sufficient to wash out the target compounds.

\subsection{Identification of $\mathrm{DHC}$}

The molecular ion $[\mathrm{M}]^{+}$of $\mathrm{DHC}$ in the positive mode was observed at $\mathrm{m} / \mathrm{z} 366$, corresponding to the cationic form of DHC. The fragment ions at $m / z 351,350,334,322$ and 308 were assigned as $\left[\mathrm{M}-\mathrm{CH}_{3}\right]^{+},\left[\mathrm{M}-\mathrm{CH}_{3}-\mathrm{H}\right]^{+},\left[\mathrm{M}-\mathrm{CH}_{3}-\mathrm{H}-\mathrm{CH}_{4}\right]^{+},\left[\mathrm{M}-\mathrm{CH}_{3}-\mathrm{H}-\mathrm{CO}\right]^{+}$and $\left[\mathrm{M}-\mathrm{CH}_{3}-\right.$ $\mathrm{CH}_{3}-\mathrm{CO}^{+}$, respectively. Moreover, $m / z$ at 290 was fragmented by dehydrogenation [11] and loss of $\mathrm{CH}_{4}$ [12] from the fragment ion with $m / z$ 308. The fragment ion with $m / z 278$ was also generated by the loss of $\mathrm{CH}_{3} \mathrm{CH}_{3}$ from the ion $m / z$ 308. The fragmentation scheme for DHC is proposed in Figure 1. 
A $=+\mathrm{EPI}(366.30)$ Charge(+0)CE(38)FT(3.1862): Exp 2, 28.194 to 28.199... Max. 6.4e7 cps.
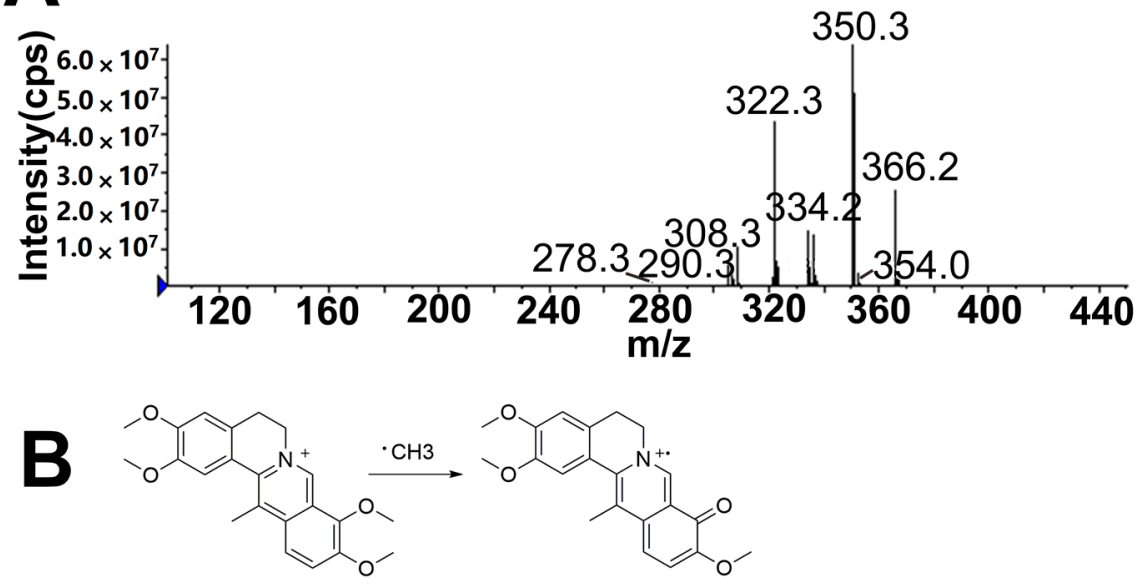

366<smiles></smiles>
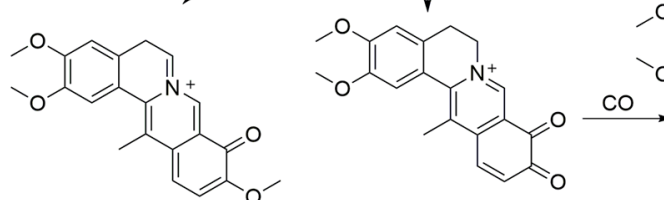<smiles>COc1cc2c(cc1OC)-c1c(C)c3c(c[n+]1CC2)C(=O)C=C3</smiles>

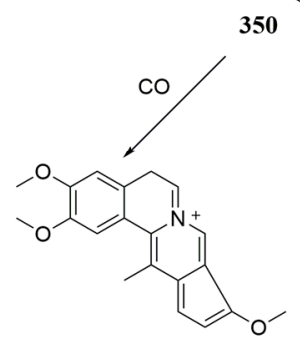

322

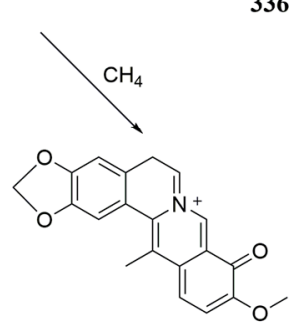

334
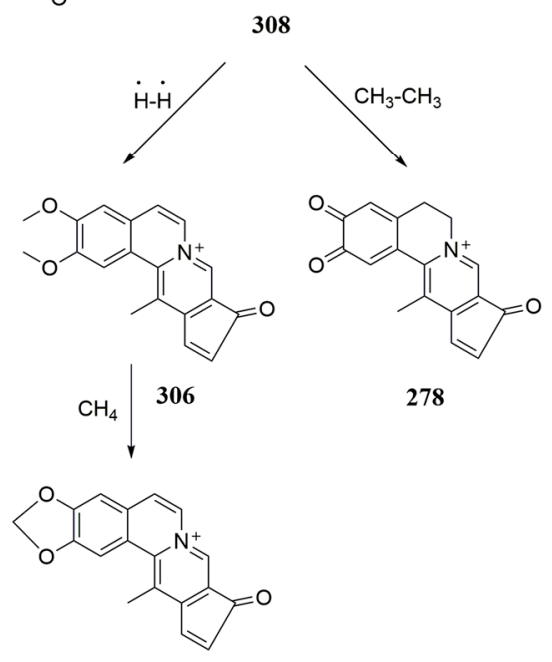

290

Figure 1. Mass spectrometry $\left(\mathrm{MS}^{2}\right)$ spectrum (A) of dehydrocorydaline (DHC) and its proposed fragmentation pathways $(\mathbf{B})$.

\subsection{Identification of Metabolites}

On the basis of the generic biotransformation table built in Metabolite ID (MetID), 49 MRM transitions were monitored during the complete analytical run. Once a listed metabolite was found by MRM, the EPI spectral acquisition of the metabolite was triggered. A total of 18 metabolites detected in rat bile and plasma are listed in Table 1. The MRM extracted ion chromatograms (XICs) of the metabolites of DHC in bile and plasma are shown in Figures 2 and 3. Compared with the peaks in the corresponding blank sample, 18 additional peaks in rat bile and 9 in plasma were observed and presumed to be metabolites. The observed metabolites were generated by $\mathrm{O}$-demethylation, $\mathrm{O}$-demethylation followed by glucuronidation, $\mathrm{O}$-demethylation followed by sulfation, di-hydroxylation and dehydrogenation, di-hydroxylation and hydroxylation. 
Table 1. Mass spectrometry $\left(\mathrm{MS}^{2}\right)$ data of dehydrocorydaline (DHC) and its metabolites.

\begin{tabular}{ccccc}
\hline No. & $\begin{array}{c}\text { Parent } \\
\text { Ion } \boldsymbol{m} / \boldsymbol{z}\end{array}$ & $\begin{array}{c}\text { Retention } \\
\text { Time (min) }\end{array}$ & Formula & Product Ions (MS ${ }^{\mathbf{2}}$ ) \\
\hline DHC & 366 & 28.4 & $\mathrm{C}_{22} \mathrm{H}_{24} \mathrm{NO}_{4}{ }^{+}$ & $351,350,334,322,308,290,278$ \\
M1, M2, M3, M4 & 352 & $15.0,15.9,16.7$, & $\mathrm{C}_{21} \mathrm{H}_{22} \mathrm{NO}_{4}{ }^{+}$ & $337,336,322,309,308,293$ \\
M5, M6, M7 & 528 & $4.4,5.0,5.5$ & $\mathrm{C}_{27} \mathrm{H}_{30} \mathrm{NO}_{10}{ }^{+}$ & $352,337,322308,293$ \\
M8, M9, M10 & 432 & $10.9,12.4,16.0$ & $\mathrm{C}_{21} \mathrm{H}_{22} \mathrm{NSO}_{7}^{+}$ & $352,337,322,308,293$ \\
M11 & 382 & 7.2 & $\mathrm{C}_{22} \mathrm{H}_{24} \mathrm{NO}_{5}{ }^{+}$ & $367,366,364,352,350,338,324,321,308,305,294$ \\
M12, M13, M14 & 382 & $11.3,15.7,27.8$ & $\mathrm{C}_{22} \mathrm{H}_{24} \mathrm{NO}_{5}{ }^{+}$ & $367,366,352,350,349,338,324,321,306$ \\
M15 & 398 & 12.3 & $\mathrm{C}_{22} \mathrm{H}_{24} \mathrm{NO}_{6}{ }^{+}$ & $383,382,368,354,340,338,324,322,294,266$ \\
M16 & 398 & 13.8 & $\mathrm{C}_{22} \mathrm{H}_{24} \mathrm{NO}_{6}{ }^{+}$ & $383,382,380,368,366,365,354,340,337$ \\
M17 & 396 & 4.7 & $\mathrm{C}_{22} \mathrm{H}_{22} \mathrm{NO}_{6}{ }^{+}$ & $381,380,378,366,364,352,350,338,335,322$ \\
M18 & 396 & 7.7 & $\mathrm{C}_{22} \mathrm{H}_{22} \mathrm{NO}_{6}{ }^{+}$ & $381,380,366,364,352,350,337,336,332$ \\
\hline
\end{tabular}

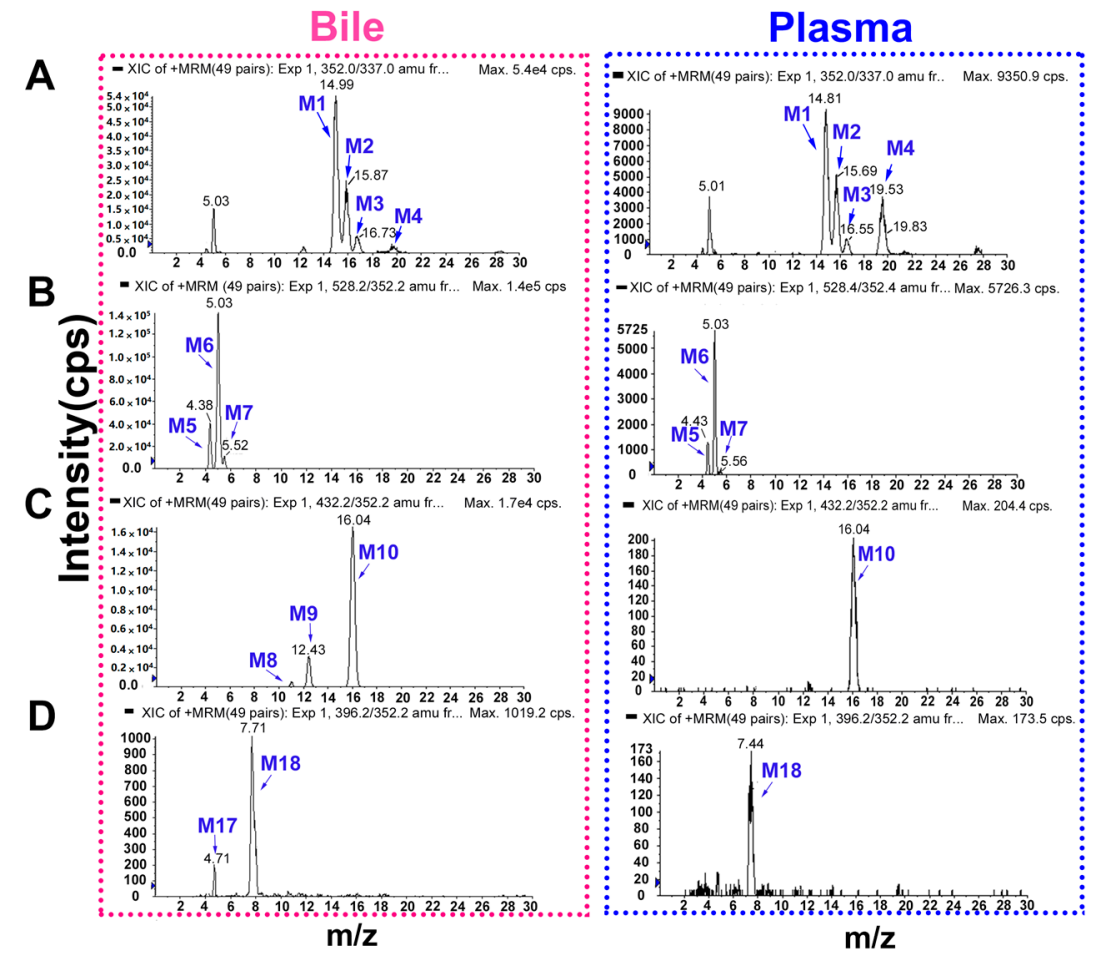

Figure 2. The extracted ion chromatograms (XICs) of the dehydrocorydaline (DHC) metabolites in bile and plasma. (A) O-demethylated metabolites (M1-M4); (B) O-demethylated metabolites followed by glucuronidation (M5-M7); (C) O-demethylated metabolites followed by sulfation (M8-M10); and (D) metabolites due to di-hydroxylation and dehydrogenation (M17 and M18).
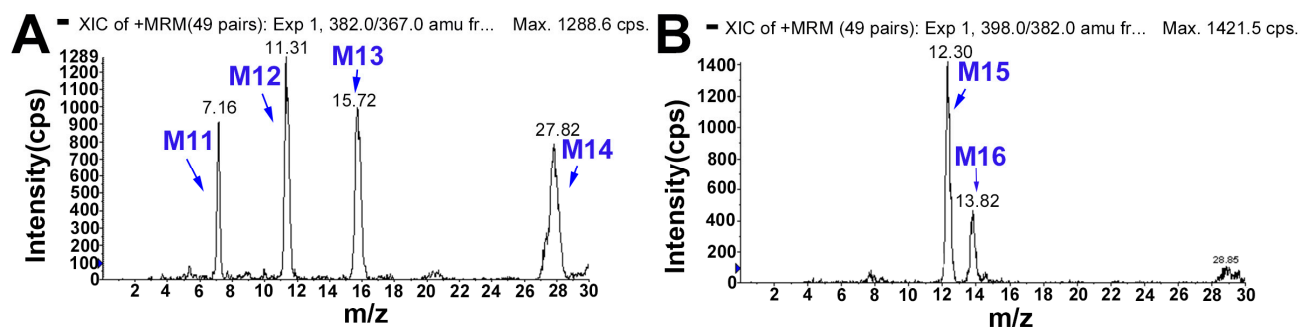

Figure 3. The extracted ion chromatograms (XICs) of dehydrocorydaline (DHC) metabolites in bile. (A) Hydroxylated metabolites, and (B) di-hydroxylated metabolites. 


\subsection{Structure Elucidation of DHC Metabolites}

\subsubsection{Metabolites Produced by O-demethylation (M1, M2, M3 and M4)}

M1, M2, M3 and M4 were detected at approximately 15.0, 15.9, 16.7 and $19.5 \mathrm{~min}$, respectively. Each of these exhibited a molecular ion at $m / z$ 352, which indicated the loss of one methyl group (14 Da) from DHC. The product ions (Figure 4 and supplementary material Figure S1) at $m / z 337$ and 336 were generated by the loss of $\mathrm{CH}_{3}(-15 \mathrm{Da})$ and $\mathrm{CH}_{4}(-16 \mathrm{Da})$ from the molecular ion. The product ions at $m / z 322$ and 309 were formed by the loss of $\mathrm{CH}_{3}(-15 \mathrm{Da})$ and $\mathrm{CO}(-28 \mathrm{Da})$ from the product ion at $m / z$ 337. The product ions at $\mathrm{m} / z 309$ further lost a molecule of $\mathrm{CH}_{4}$ to generate the product ion at $m / z$ 293. Similarly, the product ion at $m / z 336$ lost a CO fragment to yield the product ion at $m / z$ 308. To further identify the chemical position that was $O$-demethylated, the XICs of 13-methyl-dehydrocorydalmine, dehydrocorybulbine, 13-methyl-palmatrubine and palmatine were compared to those of M1-M4. The retention time $\left(t_{\mathrm{R}}\right)$ of 13-methyl-dehydrocorydalmine, dehydrocorybulbine and 13-methyl-palmatrubine were 15.1, 16.9 and $19.8 \mathrm{~min}$ (Figure S1), respectively, which matched those of M1, M3 and M4. DHC was not demethylated at C-13 in rat bile and plasma, as indicated by the absence of the signal peak of palmatine $\left(t_{R}=21.5 \mathrm{~min}\right)$ shown in Figure S1. The 2-O-methyl group of DHC was more unstable in the metabolic pathway of $O$-demethylation. $\mathrm{M} 2$ was presumed to be generated by $\mathrm{O}$-demethylation of DHC at C-2. Accordingly, M1, M2, M3 and M4 were identified as 13-methyl-dehydrocorydalmine, 13-methyl-columbamine, dehydrocorybulbine and 13-methyl-palmatrubine.
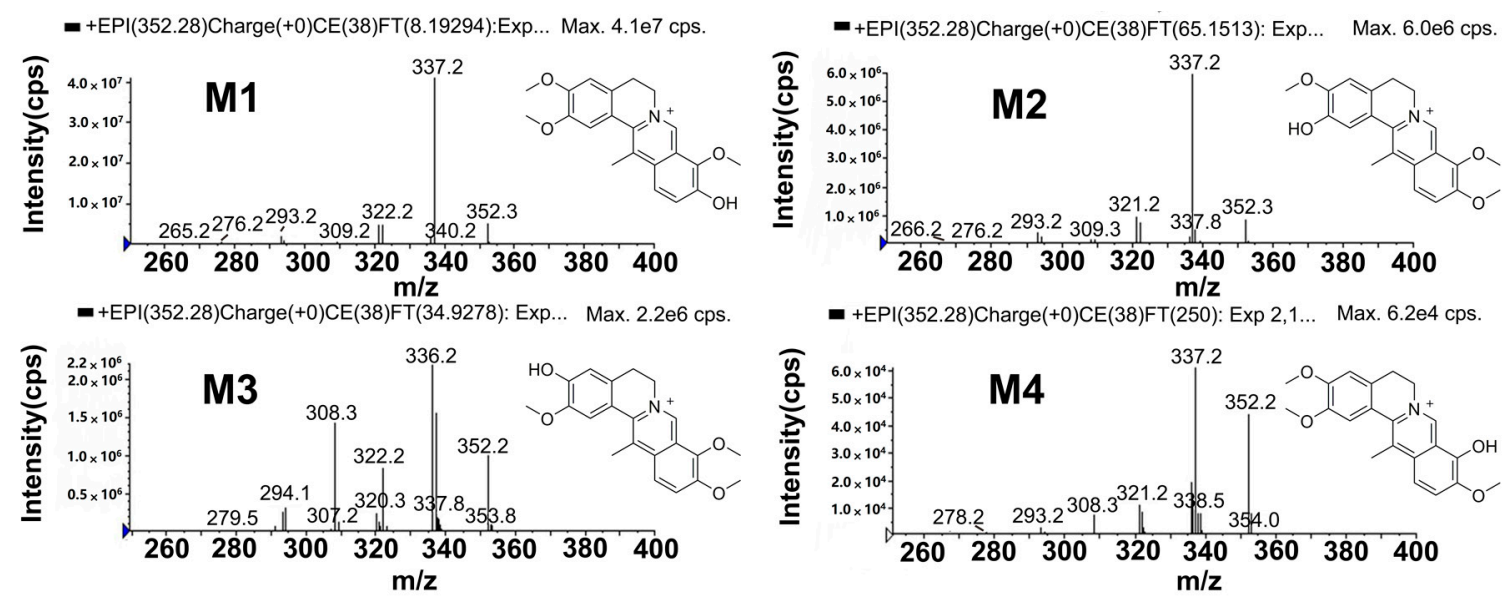

Figure 4. The mass spectrometry $\left(\mathrm{MS}^{2}\right.$ ) spectra of 13-methyl-dehydrocorydalmine (M1), 13-methylcolumbamine (M2), dehydrocorybulbine (M3) and 13-methyl-palmatrubine (M4).

\subsubsection{Metabolites Produced by Glucuronidation of O-Demethyl-DHC (M5, M6 and M7)}

M5 ( $\left.t_{\mathrm{R}}=4.4 \mathrm{~min}\right), \mathrm{M} 6\left(t_{\mathrm{R}}=5.0 \mathrm{~min}\right)$ and $\mathrm{M} 7\left(t_{\mathrm{R}}=5.5 \mathrm{~min}\right.$ ) had molecular ions (Figure 5$)$ at $\mathrm{m} / \mathrm{z}$ 528 , which were $176 \mathrm{Da}$ heavier than M1, M2, M3 or M4. The product ion at $m / z 352$, generated by the loss of $\mathrm{C}_{6} \mathrm{H}_{8} \mathrm{O}_{6}$ from the molecular ion, implied that glucuronidation occurred for M1, M2, M3 or M4. The fragmentation behaviors of M5, M6 and M7 were similar to those of the glucuronide conjugates of O-demethyl-palmatine [11]. Therefore, M5, M6 and M7 were deduced to be the glucuronide products of $O$-demethyl-DHC. 

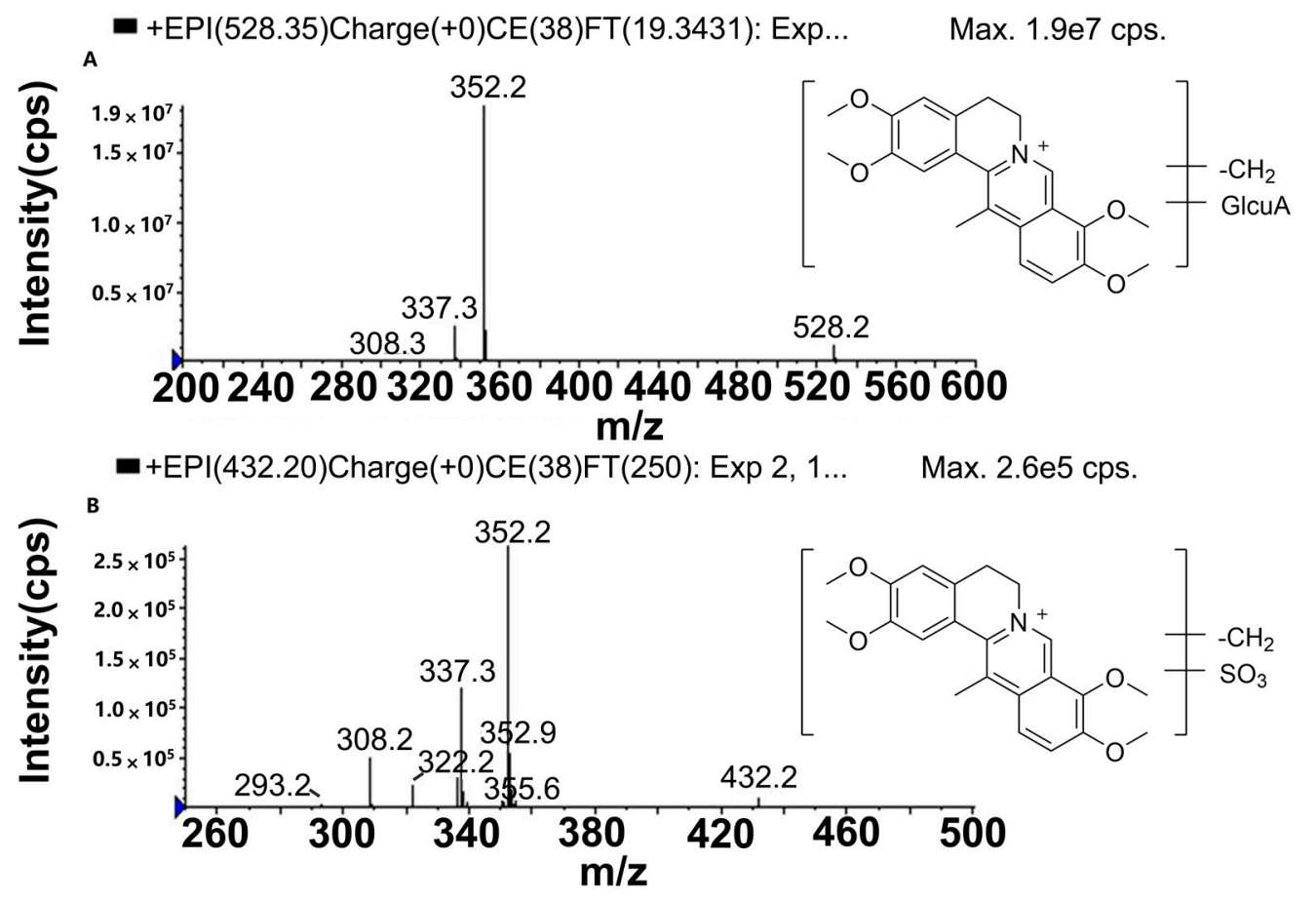

Figure 5. The mass spectrometry $\left(\mathrm{MS}^{2}\right)$ spectra of $\mathrm{O}$-demethylated metabolites followed by glucuronidation (A: M5, M6 and M7) and sulfation (B: M8, M9 and M10).

\subsubsection{Metabolites Produced by Sulfation of O-demethyl-DHC (M8, M9 and M10)}

M8 and M9 detected in bile and M10 detected in both bile and plasma were eluted at $t_{\mathrm{R}}$ values of 10.9, 12.4 and $16.0 \mathrm{~min}$, respectively. Each of these exhibited a molecular ion at $m / z 432$ (Figure 5), which was increased by 80 Da compared with M1, M2 or M3. The molecular ion at $m / z 432$ produced five major product ions at $m / z 352,337,322,308$ and 293. The product ion at $m / z 352$ was formed by the loss of $\mathrm{SO}_{3}$ from the molecular ion, and further lost a $\mathrm{CH}_{3}$ group to generate the product ion at $m / z$ 337. The product ion at $m / z 322$ originated from the loss of a $\mathrm{CH}_{3}$ group by the product ion at $m / z$ 337. The product ion at $m / z 308$ was ascribed to $\left[\mathrm{M}-\mathrm{SO}_{3}-\mathrm{CH}_{3}-\mathrm{H}-\mathrm{CO}\right]^{+}$, from which the ion with $m / z 293$ was formed through the loss of a $\mathrm{CH}_{3}$ group. The five product ions mentioned above were present in the MS/MS spectrum of M1, M2, M3 and M4. The molecular and fragment ions of M8, M9 and M10 were 14 Da higher than the corresponding ions of sulfated conjugates of O-demethyl-palmatine [13]. Accordingly, M8, M9 and M10 were assigned as the sulfated conjugates of O-demethyl-DHC.

\subsubsection{Metabolites Produced by Hydroxylation (M11, M12, M13 and M14)}

M11, M12, M13 and M14 in rat bile were eluted at retention times of 7.2, 11.3, 15.7 and $27.8 \mathrm{~min}$, and all displayed the molecular ion at $m / z 382$, which was 16 Da higher than that of DHC and $14 \mathrm{Da}$ higher than that of mono-hydroxylated metabolites of palmatine [14]. The product ions (Figure 6 and Supplementary Figure S2), specifically at $m / z 367\left(\left[\mathrm{M}-\mathrm{CH}_{3}\right]^{+}\right), 366\left(\left[\mathrm{M}-\mathrm{CH}_{3}-\mathrm{H}\right]^{+}\right), 350([\mathrm{M}-$ $\left.\left.\mathrm{CH}_{3}-\mathrm{H}-\mathrm{CH}_{4}\right]^{+}\right), 338\left(\left[\mathrm{M}-\mathrm{CH}_{3}-\mathrm{H}-\mathrm{CO}\right]^{+}\right)$and $324\left(\left[\mathrm{M}-\mathrm{CH}_{3}-\mathrm{CH}_{3}-\mathrm{CO}\right]^{+}\right)$, were also 16 Da higher than the corresponding fragment ions of DHC. These results indicated that M11, M12, M13 and M14 may have been the mono-hydroxylated products of DHC. In addition, the product ion of M11 at $m / z 364\left(\left[\mathrm{M}-\mathrm{H}_{2} \mathrm{O}\right]^{+}\right)$and those of M12, M13 and M14 at $m / z 349\left(\left[\mathrm{M}-\mathrm{CH}_{3}-\mathrm{H}_{2} \mathrm{O}\right]^{+}\right)$, $321\left(\left[\mathrm{M}-\mathrm{CH}_{3}-\mathrm{H}_{2} \mathrm{O}-\mathrm{CO}\right]^{+}\right)$and $306\left(\left[\mathrm{M}-\mathrm{CH}_{3}-\mathrm{H}_{2} \mathrm{O}-\mathrm{CO}-\mathrm{CH}_{3}\right]^{+}\right)$indicated that $[\mathrm{M}]^{+}$ underwent dehydration between $\mathrm{C}-5$ and -6 . Therefore, M11, M12, M13 and M14 were identified as 5- or 6-hydroxyl-DHC and its stereoisomer. 


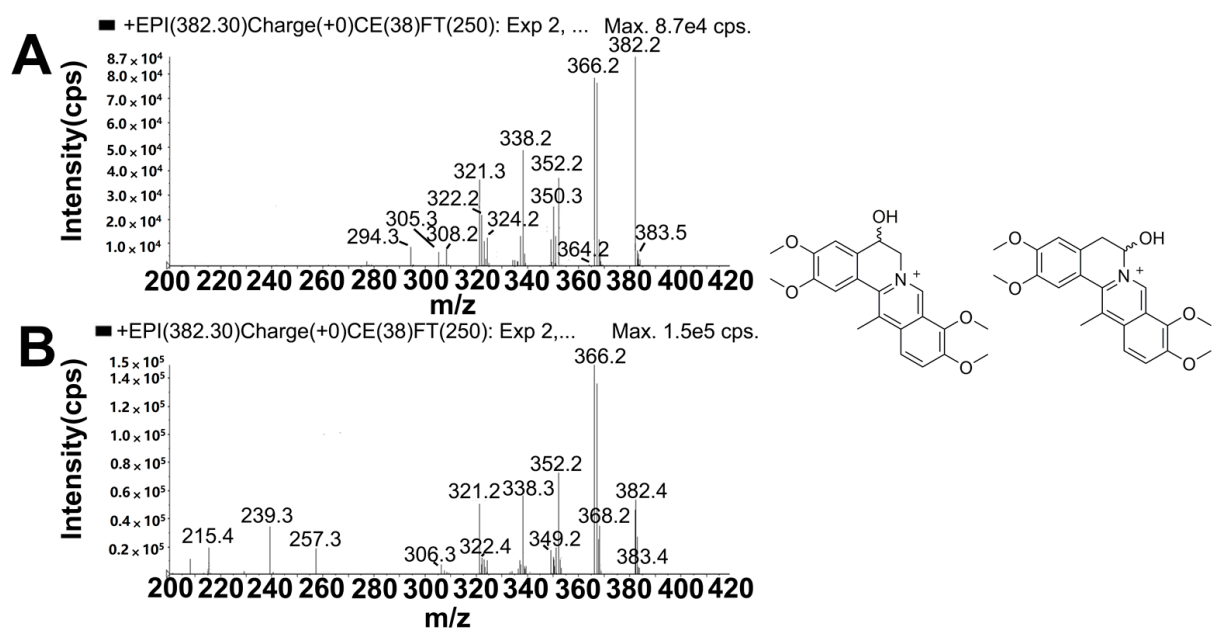

Figure 6. The mass spectrometry $\left(\mathrm{MS}^{2}\right)$ spectra of hydroxylated metabolites (A: M11; B: M12, M13 and M14).

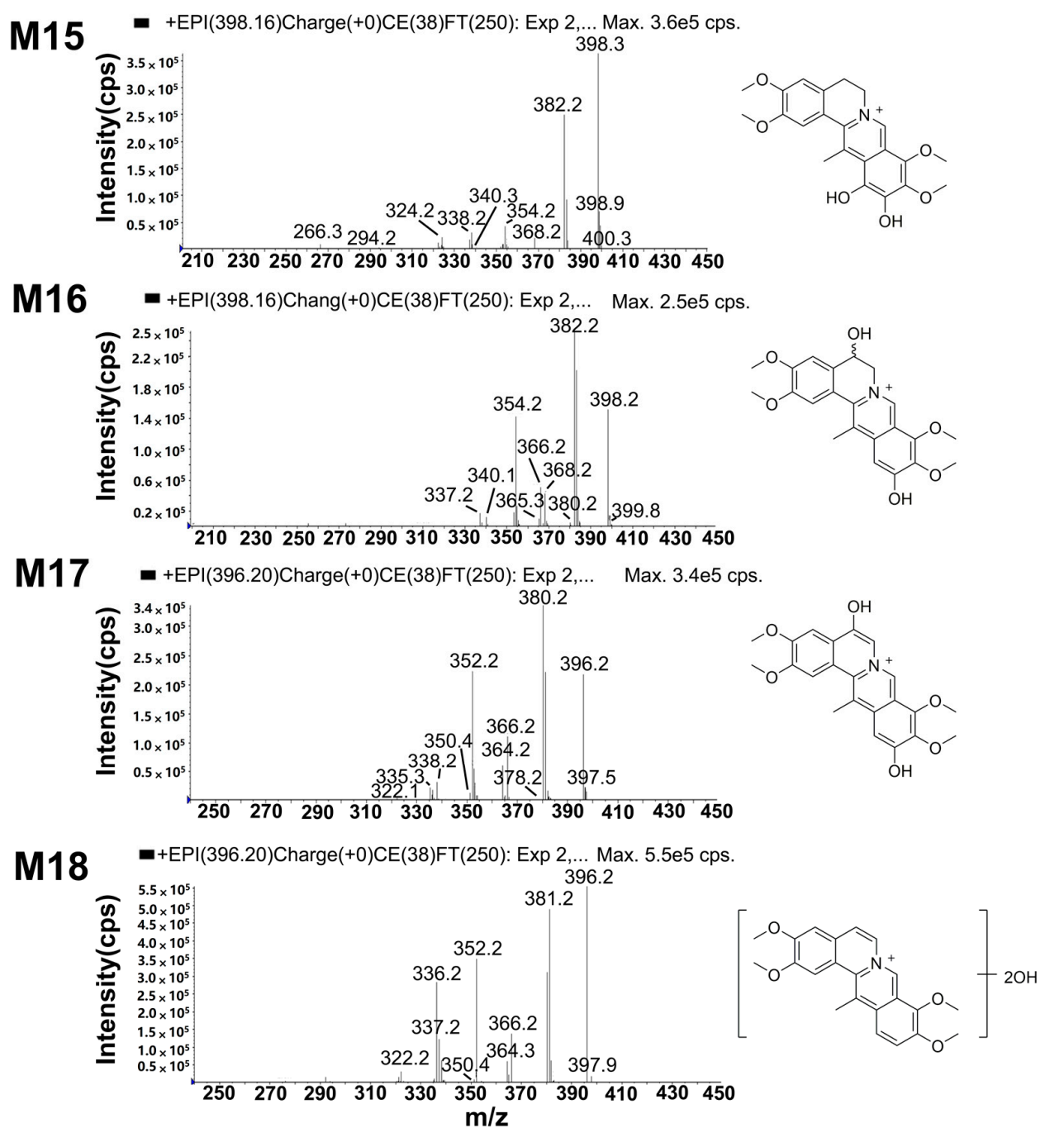

Figure 7. The mass spectrometry $\left(\mathrm{MS}^{2}\right)$ spectra of di-hydroxylated metabolites (M15 and M16) and metabolites (M17 and M18) produced by di-hydroxylation and dehydrogenation. 


\subsubsection{Metabolites Produced by Di-Hydroxylation (M15 and M16)}

Both M15 $\left(t_{\mathrm{R}}=12.3 \mathrm{~min}\right)$ and M16 ( $\left.t_{\mathrm{R}}=13.8 \mathrm{~min}\right)$ displayed a molecular ion at $\mathrm{m} / \mathrm{z}$ 398, which was 32 Da heavier than that of DHC and 14 Da heavier than that of di-hydroxylated metabolites of palmatine [15]. The fragment ions (Figure 7) at $m / z 383\left(\left[\mathrm{M}-\mathrm{CH}_{3}\right]^{+}\right), 382\left(\left[\mathrm{M}-\mathrm{CH}_{4}\right]^{+}\right), 354([\mathrm{M}-$ $\left.\left.\mathrm{CH}_{3}-\mathrm{H}-\mathrm{CO}\right]^{+}\right)$and $340\left(\left[\mathrm{M}-\mathrm{CH}_{3}-\mathrm{CH}_{3}-\mathrm{CO}^{+}\right.\right.$were increased by $32 \mathrm{Da}$ compared with those of DHC. Thus, M15 and M16 were identified as the di-hydroxylates of DHC. Moreover, the fragment ions of M16 (Figure S3) at $m / z 380,365$ and 337 were assigned as $\left[\mathrm{M}-\mathrm{H}_{2} \mathrm{O}\right]^{+},\left[\mathrm{M}-\mathrm{H}_{2} \mathrm{O}-\mathrm{CH}_{3}\right]^{+}$and $\left[\mathrm{M}-\mathrm{H}_{2} \mathrm{O}-\mathrm{CH}_{3}-\mathrm{CO}^{+}\right.$, which indicated that M16 underwent dehydration to generate a double bond between $\mathrm{C}-5$ and -6 . These results implied that one of the hydroxylation positions of M16 was either C -5 or -6 . According to a previous report [14], the position order of a hydroxylation reaction is $\mathrm{C}-5,-11$ and -6 ; the hydroxylation of $\mathrm{C}-5$ is easier than that of the other positions. M16 was tentatively identified as 5,11-di-hydroxyl-DHC. The fragment ions of $\mathrm{M} 15$ at $m / z 338\left(\left[\mathrm{M}-2 \mathrm{CH}_{3}-\mathrm{CO}-2 \mathrm{H}\right]^{+}\right)$, $322\left(\left[\mathrm{M}-2 \mathrm{CH}_{3}-\mathrm{CO}-2 \mathrm{H}-\mathrm{CH}_{4}\right]^{+}\right), 294\left(\left[\mathrm{M}-2 \mathrm{CH}_{3}-\mathrm{CO}-\mathrm{CH}_{4}-2 \mathrm{H}-\mathrm{CO}\right]^{+}\right)$and $266([\mathrm{M}-$ $\left.2 \mathrm{CH}_{3}-\mathrm{CO}-\mathrm{CH}_{4}-2 \mathrm{H}-2 \mathrm{CO}\right]^{+}$) showed the loss of two hydrogen atoms, a characteristic feature of the collision spectrum of M15, which indicated that the two hydroxyl modifications occurred at the D-ring. The proposed fragmentation pathway is shown in Figure S4. The first hydroxyl group at C-11 underwent arrangement to saturate C-12 adjacent to the aromatic $\left(\mathrm{sp}^{2}\right)$ carbon atom, which made C-12 easier to be oxidized (Figure 8).

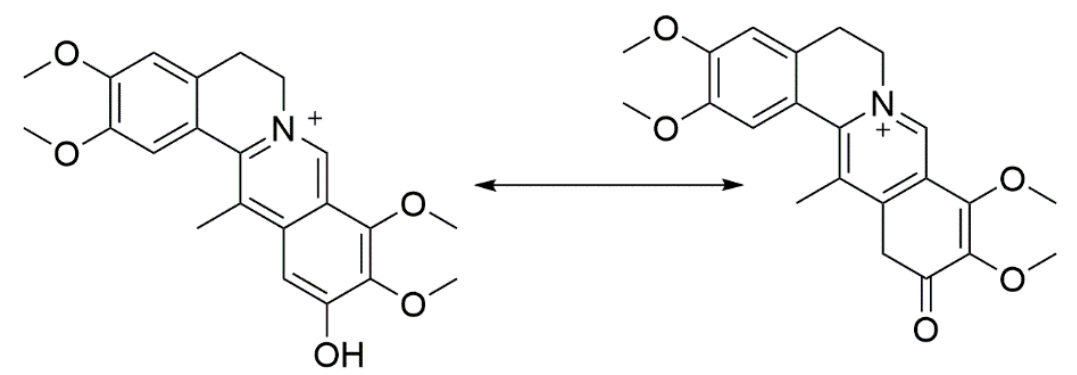

Figure 8. The arrangement of 11-hydroxyl dehydrocorydaline (DHC).

\subsubsection{Metabolites Produced by Di-Hydroxylation and Dehydrogenation (M17 and M18)}

M17 in bile and M18 in both bile and plasma were eluted at approximately 4.7 and $7.7 \mathrm{~min}$, respectively. Each of these exhibited a molecular ion at $m / z 396$ (Figure 7), which was 2 Da lighter than M15 or M16. The results indicated that dehydrogenation had occurred to M17 and M18. M17 and M18 were deduced to be dehydrogenated products of di-hydroxyl-DHC. The fragment ions of M17 at $m / z 380,366,364,352$ and 338 were 2 Da less than the corresponding fragment ions of M16. Moreover, the fragment ions of M17 at $m / z 378\left(\left[\mathrm{M}-\mathrm{CH}_{4}-\mathrm{H}_{2}\right]^{+}\right), 350\left(\left[\mathrm{M}-\mathrm{CH}_{4}-\mathrm{H}_{2}-\mathrm{CO}\right]^{+}\right)$and $335\left(\left[\mathrm{M}-\mathrm{CH}_{4}-\mathrm{H}_{2}-\mathrm{CO}-\mathrm{CH}_{3}\right]^{+}\right)$indicated that oxidation occurred at $\mathrm{C}-5$ and -11 . Accordingly, M17 was a dehydrogenation product of M16, namely, 5,11-di-hydroxylated-5,6-dehydro-DHC. Its proposed fragmentation pathway is shown in Figure S5.

\subsection{A Summary of Metabolic Pathways for DHC}

According to the above analysis results, DHC undergoes O-demethylation (M1, M2, M3 and M4), O-demethylation followed by glucuronidation (M5, M6 and M7), O-demethylation followed by sulfation (M8, M9 and M10), hydroxylation (M11, M12, M13 and M14), di-hydroxylation (M15 and M16), and di-hydroxylation and dehydrogenation (M17 and M18). In total, 18 metabolites were detected in the bile and 9 were identified in plasma. The proposed metabolic pathways of DHC is shown in Figure 9. 


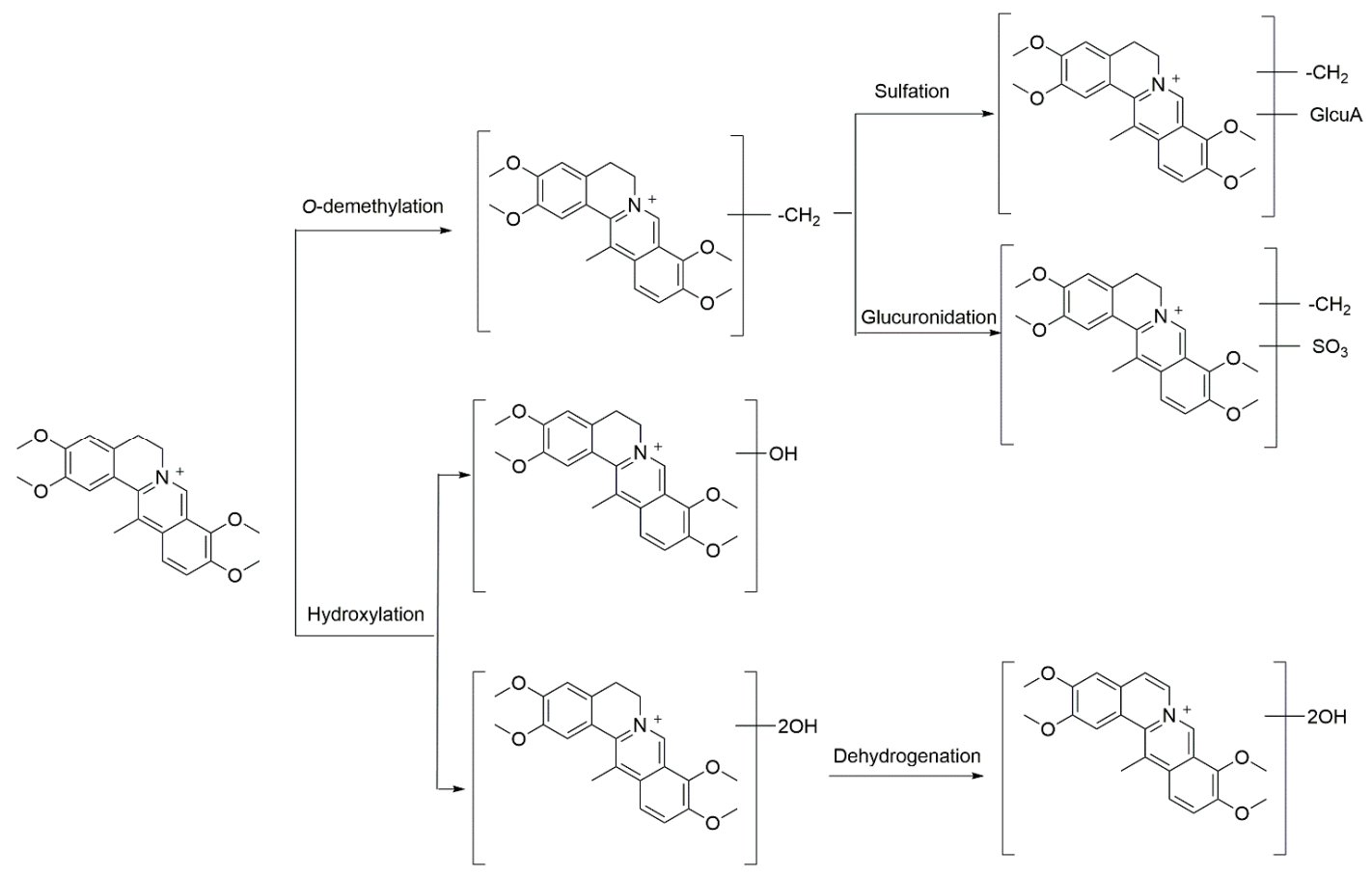

Figure 9. Proposed metabolic pathways of dehydrocorydaline (DHC) in rats.

\section{Experimental Section}

\subsection{Materials and Reagents}

The standard references DHC, 13-methyl-dehydrocorydalmine, dehydrocorybulbine, 13-methylpalmatrubine and palmatine were isolated and purified from the dried tuber of $C$. yanhusuo in the authors' laboratory. The purity of the isolated compounds was found to be above $98.0 \%$ as determined by HPLC with a photodiode array detector. HPLC-grade acetonitrile and methanol were purchased from Honeywell Burdick \& Jackson Company (Mexico City, Mexico). Formic acid (LC-MS grade) was purchased from Fisher Scientific (Thermo Fisher, Geel, Belgium). Ammonium acetate (HPLC-grade) was purchased from ROE Scientific Inc. (Newark, NJ, USA). Water was deionized using a Milli-Q water purification system (Millipore, Milford, MA, USA). All other reagents were of analytical grade.

\subsection{Instruments and LC-MS/MS Conditions}

An Agilent 1100 system (Agilent Technologies, Santa Clare, CA, USA), consisting of a vacuum degasser, a quaternary pump, a column oven and an auto-sampler, was used coupled to an API 3200 QTRAP mass spectrometer (Applied Biosystems/MDS SCIEX, Concord, ON, Canada). The MetID database (version 1.4.1) was from Applied Biosystems.

Chromatographic separation was achieved on a Diamonsil C18 analytical column (4.6 $\mathrm{mm} \times 250 \mathrm{~mm}, 5 \mu \mathrm{m}$; Dikma Corporation, Beijing, China) eluted with a mobile phase of acetonitrile and water (containing $0.8 \%$ formic acid and $10 \mathrm{mM}$ ammonium acetate; $25: 75 \mathrm{v} / \mathrm{v}$ ) at a flow rate of $1 \mathrm{~mL} / \mathrm{min}$. The column temperature was set at $25^{\circ} \mathrm{C}$ and the injection volume was $30 \mu \mathrm{L}$.

The MS parameters were as follows: ESI was performed in the positive ionization mode; curtain gas (CUR): 10.0 psi; collision gas (CAD): medium; ion spray (IS) voltage: $5500 \mathrm{~V}$; source temperature: $450{ }^{\circ} \mathrm{C}$; GS1: 70 psi and GS2: 60 psi; declustering potential (DP): $70 \mathrm{~V}$; collision energy (CE): $32 \mathrm{eV}$; entrance potential (EP): $10 \mathrm{~V}$; collision cell exit potential (CXP): $3 \mathrm{~V}$. When MRM-IDA-EPI was performed, the IDA threshold was set at 200 counts per second and the CE in EPI mode was set at $38 \mathrm{eV}$ (CE spread of $15 \mathrm{eV}$ ). The EPI scan was operated at a scan rate of $4000 \mathrm{amu} / \mathrm{s}$ with dynamic fill in the linear ion trap. 


\subsection{Animal Handling}

Male Sprague-Dawley (SD) rats (140-160 g) were obtained from the Department of Laboratory Animal Resources at the National Institutes for Food and Drug Control (certification number: SCXK2014-0013; Beijing, China). The rats were kept in a controlled environment at a temperature of $20-25{ }^{\circ} \mathrm{C}$ and a relative humidity of $40-60 \%$ on a standard $12 / 12 \mathrm{hlight} /$ dark cycle with free access to water and a normal diet for 7 days. All the experiments were performed according to the National Institutes of Health Guidelines for Animal Research and were approved by the Ethics Committee of the Institute of Medicinal Plant Development, CAMS \& PUMC.

For the bile samples, six rats were fasted overnight with free access to water before the test. The rats were anesthetized with $10 \%(w / v)$ chloral hydrate solution (at a dose of $3.0 \mathrm{~mL} / \mathrm{kg}$ ) by intraperitoneal injection and were fixed on a thermo-controlled surgery platform. The bile duct of the rats was cannulated with a polyethylene tube (PE-10) to collect bile samples continuously, as previously described [16]. The blank bile samples were collected within $2 \mathrm{~h}$ of bile duct cannulation. The bile samples were collected within $12 \mathrm{~h}$ of oral administration of DHC at a dose of $97.5 \mathrm{mg} / \mathrm{kg}$. All the bile samples were stored at $-80^{\circ} \mathrm{C}$ until analysis.

For the plasma sample, nine rats were fasted overnight, but allowed water ad libitum. Six of the rats were orally administered DHC $(97.5 \mathrm{mg} / \mathrm{kg}, 10 \mathrm{~mL} / \mathrm{kg})$. The remaining three rats received the corresponding volume of blank vehicle to obtain the blank plasma. The blood samples (about $0.3 \mathrm{~mL}$ ) from the retro-orbital plexus were collected into heparinized tubes at $0.25,0.5,0.75,1,2,4,6$ and $8 \mathrm{~h}$ after dosing and were then centrifuged at $3000 \mathrm{rpm}$ for $10 \mathrm{~min}$. The obtained plasma samples were transferred to an Eppendorf tube and stored at $-80^{\circ} \mathrm{C}$ for subsequent analysis.

\subsection{Sample Preparation}

Bile samples were thawed at room temperature and centrifuged at $9000 \mathrm{rpm}$ for $10 \mathrm{~min}$. The supernatant was loaded onto a pre-conditioned OASIS HLB column ( $30 \mathrm{mg}, 30 \mu \mathrm{m}, 1 \mathrm{~mL}$; Waters, Milford, MA, USA). Then the SPE column was eluted with $1 \mathrm{~mL}$ of water and $1 \mathrm{~mL}$ of methanol (containing $2 \%$ formic acid, $v / v$ ) in succession. The methanol fraction was collected and evaporated under a gentle flow of nitrogen at $40{ }^{\circ} \mathrm{C}$. The residue was reconstituted in $150 \mu \mathrm{L}$ of $10 \%$ acetonitrile and centrifuged at 12,000 rpm for $10 \mathrm{~min}$. A $30 \mu \mathrm{L}$ volume of the supernatant was injected into the HPLC-MS/MS system for analysis.

The plasma samples were pooled together to generate a single sample of each matrix. Then, the mixed plasma samples were processed in a similar manner as the bile samples.

\section{Conclusions}

In this study, a LC-MS/MS method was established to detect the metabolites of DHC in plasma and bile samples of rats after oral administration of DHC, an active alkaloid from C. yanhusuo used in the treatment of cardiovascular disease. A total of 18 metabolites were identified using the MRM-IDA-EPI mode, and their structures were elucidated on the basis of the retention times of reference standards and characteristic fragment ions. The main metabolic pathways were $\mathrm{O}$-demethylation, $\mathrm{O}$-demethylation followed by glucuronidation, $\mathrm{O}$-demethylation followed by sulfation, di-hydroxylation and dehydrogenation, di-hydroxylation and hydroxylation. The experimental results are useful for further investigation of the biotransformation of DHC in other biological matrices and the metabolism of other quaternary protoberberine-type alkaloids. Moreover, our findings provide valuable information for pharmacological research of DHC.

Supplementary Materials: Supplementary materials are available online. Figure S1: The XICs of reference standards (A): (a) 13-methyl-dehydrocorydalmine, (b) dehydrocorybulbine, (c) 13-methyl-palmatrubine and (d) palmatine. Proposed fragmentation pathways (B) for M1, M2, M3 and M4. Figure S2: Proposed fragmentation pathways for M11, M12, M13 and M14. Figure S3: Proposed fragmentation pathways for M16. Figure S4: Proposed fragmentation pathways for M15. Figure S5: Proposed fragmentation pathways for M17. 
Acknowledgments: This work was supported by the National Natural Science Foundation of China (Grant No. 30772710), the Science and Technology Major Programmer for Major Drug Discovery (Grant No. 2012ZX09301002-001-028) and the CAMS Innovation Fund for Medical Sciences (CIFMS; Grant No. 2016-I2M-1-012).

Author Contributions: Y. Shi and H. Guan participated in research design. H. Guan, K. Li, X. Wang, X. Luo, M. Su, W. Tan, and X. Chang were responsible for performing the experiments. Y. Shi, H. Guan and K. Li performed data analysis. Y. Shi and H. Guan contributed to the writing and editing of the manuscript.

Conflicts of Interest: The authors declare no conflicts of interest.

\section{References}

1. Ling, H.; Wu, L.; Li, L. Corydalis yanhusuo rhizoma extract reduces infarct size and improves heart function during myocardial ischemia/reperfusion by inhibiting apoptosis in rats. Phytother. Res. PTR 2006, 20, 448-453. [CrossRef] [PubMed]

2. Hu, J.N.; Zhai, L.; Zhang, Q.M.; Jiang, M.X.; Ye, W.; Wang, R.W. To observe the clinical efficacy and safety on treating coronary heart disease and angina pectoris with kodaling tablets. Clin. J. Chin. Med. 2014, 6, 31-34.

3. Xu, Z.; Chen, X.; Fu, S.; Bao, J.; Dang, Y.; Huang, M.; Chen, L.; Wang, Y. Dehydrocorydaline inhibits breast cancer cells proliferation by inducing apoptosis in mcf-7 cells. Am. J. Chin. Med. 2012, 40, 177-185. [CrossRef] [PubMed]

4. Grycova, L.; Dostal, J.; Marek, R. Quaternary protoberberine alkaloids. Phytochemistry 2007, 68, 150-175. [CrossRef] [PubMed]

5. Jiang, X.R.; Wu, Q.X.; Shi, H.L.; Chen, W.P.; Chang, S.Q.; Zhao, S.Y.; Tian, X.Y.; Zhou, L.F.; Guo, S.M.; Li, Y.J Pharmacological actions of dehydrocorydaline on cardiovascular system. Acta Pharm. Sin. 1982, 17, 61-65.

6. Li, Q.; Guan, H.; Wang, X.; He, Y.; Sun, H.; Tan, W.; Luo, X.; Su, M.; Shi, Y. Fingerprint-efficacy study of the quaternary alkaloids in corydalis yanhusuo. J. Ethnopharmacol. 2017, 207, 108-117. [CrossRef] [PubMed]

7. Peng, Y.; Li, C.; Liu, X. Determination of dehydrocorydaline content in kedaling tablets by hplc. J. Hunan Univ. Chin. Med. 2012, 32, 37-39.

8. Fujii, T.; Miyazaki, H.; Nambu, K.; Kagemoto, A.; Hashimoto, M. Disposition and metabolism of 14c-dehydrocorydaline in mice and rats. Radioisotopes 1984, 33, 519-525. [CrossRef] [PubMed]

9. Li, K.; Xing, D.; Jin, W.; Sun, H.; Shi, Y. Chemical fingerprint and metabolic fingerprint analysis of the medicinal extract of corydalis yanhusuo by hplc-uv and hplc-ms methods. Asian J. Chem. 2011, 23, 723-728.

10. Li, Q.Y.; Li, K.T.; Sun, H.; Jin, W.; Shi, J.W.; Shi, Y. Lc-ms/ms determination and pharmacokinetic study of dehydrocorydaline in rat plasma after oral administration of dehydrocorydaline and corydalis yanhusuo extract. Molecules 2014, 19, 16312-16326. [CrossRef] [PubMed]

11. Vrba, J.; Papouskova, B.; Pyszkova, M.; Zatloukalova, M.; Lemr, K.; Ulrichova, J.; Vacek, J. Metabolism of palmatine by human hepatocytes and recombinant cytochromes p450. J. Pharm. Biomed. Anal. 2015, 102, 193-198. [CrossRef] [PubMed]

12. Wang, D.; Liu, Z.; Guo, M.; Liu, S. Structural elucidation and identification of alkaloids in rhizoma coptidis by electrospray ionization tandem mass spectrometry. J. Mass Spectrom. 2004, 39, 1356-1365. [CrossRef] [PubMed]

13. Li, Y.; Wang, H.; Si, N.; Ren, W.; Han, L.; Xin, S.; Zuo, R.; Wei, X.; Yang, J.; Zhao, H.; et al. Metabolic profiling analysis of berberine, palmatine, jatrorrhizine, coptisine and epiberberine in zebrafish by ultra-high performance liquid chromatography coupled with ltq orbitrap mass spectrometer. Xenobiot. Fate Foreign Compd. Biol. Syst. 2015, 45, 302-311. [CrossRef] [PubMed]

14. Yang, Q.C.; Wu, W.H.; Han, F.M.; Chen, Y. Identification of in vivo and in vitro metabolites of palmatine by liquid chromatography-tandem mass spectrometry. J. Pharm. Pharmacol. 2009, 61, 647-652. [CrossRef] [PubMed]

15. Zhu, M.; Han, F.; Chen, H.; Peng, Z.; Chen, Y. Identification of palmatine and its metabolites in rat urine by liquid chromatography/tandem mass spectrometry. Rapid Commun. Mass Spectrom. RCM 2007, 21, 2019-2022. [CrossRef] [PubMed] 
16. Matsukawa, N.; Matsumoto, M.; Hara, H. High biliary excretion levels of quercetin metabolites after administration of a quercetin glycoside in conscious bile duct cannulated rats. Biosci. Biotechnol. Biochem. 2009, 73, 1863-1865. [CrossRef] [PubMed]

Sample Availability: Samples of the compounds are not available from the authors.

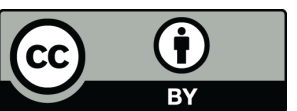

(C) 2017 by the authors. Licensee MDPI, Basel, Switzerland. This article is an open access article distributed under the terms and conditions of the Creative Commons Attribution (CC BY) license (http:/ / creativecommons.org/licenses/by/4.0/). 